\title{
Sphingosine Kinase 1: A Potential Therapeutic Target In Pulmonary Arterial
}

\section{Hypertension?}

\author{
Nigel J. Pyne* and Susan Pyne
}

Strathclyde Institute of Pharmacy and Biomedical Sciences, University of Strathclyde, 161 Cathedral St, Glasgow, G4 ORE, Scotland, UK.

* To whom correspondence should be addressed (‥j.pyne@strath.ac.uk)

Key Words: Sphingosine 1-phosphate, Sphingosine 1-phosphate receptors, Vasoconstriction, Vascular remodeling, Endothelium, Vascular smooth muscle.

\begin{abstract}
Sphingosine kinase 1 (SphK1) knockout mice are protected against pulmonary hypertension and expression levels of the enzyme are increased in the lungs of pulmonary arterial hypertensive (PAH) patients. Moreover, sphingosine 1-phosphate can promote vascular remodeling/vasoconstriction in rodent and human pulmonary arterial smooth muscle cell models. Therefore, SphK1 might be a novel target for treatment of PAH. However, in our opinion more refined strategies to target SphK1 are needed because this enzyme is protective against endothelial dysfunction and can become resistant to SphK1 inhibitors in vascular smooth muscle, thereby potentially limiting effectiveness in $\mathrm{PAH}$. In addition, SphK1 is involved in maladaptive hypertrophy and we propose that heart failure might be an additional direct target for therapeutic intervention with SphK1 inhibitors.
\end{abstract}




\section{Pulmonary Arterial Hypertension and Sphingosine 1-Phosphate}

Pulmonary arterial hypertension (PAH) affects $15-50$ people/million and is characterised by elevated mean arterial blood pressure, shortness of breath, fatigue, dizziness, chest pain and edema. PAH can lead to reduced blood flow to the heart, cardiac hypertrophy and heart failure. PAH affects small pre-capillary arteries in the lung where there is enhanced vasoconstriction and vascular remodelling (see Glossary) with an underlying inflammation [1]. Treatment is aimed at enhancing pulmonary hemodynamics, exercise tolerance and life quality. Drugs used to treat PAH are vasodilators including prostacyclin analogues, endothelin receptor antagonists and phosphodiesterase 5 inhibitors. Combination therapies with these agents has significantly improved life expectancy from $\sim 3$ years at diagnosis to 5-7 years [1]. Nevertheless progressive vascular and ventricular remodeling still occurs leading to cardiac hypertrophy/heart failure and death. Therefore, there is still an unmet medical need and recent findings suggest that sphingosine 1-phosphate (S1P) might be a potential novel target for clinical intervention in PAH. This is exemplified by the role of sphingosine kinase 1 (SphK1, which catalyses the formation of $\mathrm{S} 1 \mathrm{P}$ ) and the $\mathrm{S}_{1} \mathrm{P}_{2}$ receptor (which is specifically activated by binding of S1P) in vascular remodeling and vasoconstriction in rodent models (Figure 1). In addition, SphK1 and S1P are linked with signaling networks that involve gene products that are significantly up-regulated in human $\mathrm{PAH}$, such as the Receptor for Advanced Glycation End products (RAGE) [2] and Signal Transducer Activator of Transcription 3 (STAT3) [3]. The enzyme is also potentially linked with down-regulation of Bone Morphogenetic Protein Receptor type II (BMPR2) [4], where inactivating mutations in the BMPR2 gene are associated with familial hypertension [5]. The overlap of SphK1 signaling with known PAH susceptibility gene products provides additional support for the idea that the enzyme could be considered as a novel target for 
therapeutic intervention in PAH. However, targeting SphK1 might also enhance endothelial dysfunction [6], thereby potentially limiting effectiveness of SphK1 inhibitors in reducing vascular smooth muscle contractility/remodeling and blood pressure in humans. A plausible reason for this might be that SphK1 inhibitors reduce the levels of vasorelaxants released from the endothelium that function to oppose vasoconstriction/vascular remodeling. SphK1 inhibitors can also induce the proteasomal degradation of SphK1 in human pulmonary arterial smooth muscle (PASMC) cells and cancer cells [7-9], which probably underlies some of the major effects of these inhibitors. However, in certain cases, as will be discussed, SphK1 can become resistant to SphK1 inhibitors [7, 10] thereby potentially limiting the effectiveness of the latter in the treatment of PAH. To date, various data supporting a role for SphK1 has been obtained using different animal models of pulmonary hypertension, some of which reflect severe PAH (e.g. Sugen5416 rat model). Arguably data obtained using such severe models of PAH are likely more informative than models that do not fully recapitulate human disease, in terms of predicting translational potential of SphK1 inhibitors to man. In addition, studies on SphK1 and S1P, in terms of regulating vascular tone, use different vascular sources from rodents and it is recognized that there are differences between the vascular smooth muscle in the pulmonary circulation compared with the systemic circulation i.e. different developmental origin, which places some constraints on extrapolation of the findings to human PAH.

\section{Sphingosine kinase 1 and Hypertension}

The sphingosine kinases (two isoforms, SphK1 and SphK2) phosphorylate sphingosine to produce the bioactive lipid, S1P. Human and mouse SphK1 and SphK2 are encoded by distinct genes and the enzymes exhibit different subcellular localisation and 
biochemical properties to regulate both overlapping and non-overlapping signaling pathways [11]. S1P can either be exported from cells and bind to a family of five S1Pspecific $\mathrm{G}$ protein coupled receptors $\left(\mathrm{S}_{1} \mathrm{P}_{1-5}\right)[12,13]$ or can interact with intracellular proteins, such as human HDAC-1/2 [14] to induce biological and cellular regulation.

There is a growing body of evidence that clearly supports a significant role for SphK1 and S1P in the pathogenesis of hypertension. For instance, hypoxia, which induces pulmonary hypertension, increases SphK1 and SphK2 mRNA transcript levels in human PASMC [15]. Indeed, the SphK1 gene promoter has two hypoxia-inducible factor responsive elements (hypoxia-inducible factor $1 \alpha$ (HIF1 $\alpha)$ and hypoxia inducible factor $2 \alpha($ HIF2 $\alpha)$ ) [16]. Given the changes in SphK1 expression in human PASMC under chronic hypoxic conditions, we had previously proposed that this might be a contributing factor to the vascular remodeling in PAH [15]. A subsequent study reported increased SphK1 protein expression in the lungs and increased SphK1 protein and mRNA transcript levels in PASMC from patients with PAH [17]. Increased SphK1 protein expression levels were also found in the lungs of hypoxic-induced pulmonary hypertensive rats and mice compared with normoxic animals [17]. Similarly, elevated levels of S1P were found in the lungs of patients with $\mathrm{PAH}$ and in pulmonary arteries of hypoxic-induced pulmonary hypertensive rats and mice [17]. In contrast, no changes in SphK2 protein expression were detected in the lungs/PASMC from PAH patients or in hypoxic treated rodent models compared with healthy patients or normoxic animals respectively [17]. Moreover, $\operatorname{SphK1^{-/-}}$ mice were shown to exhibit lower right ventricular systolic pressure (RVSP), decreased right ventricular hypertrophy $(\mathbf{R V H})$ and less severe pulmonary vascular remodeling in response to hypoxia, while the development of pulmonary hypertension was not mitigated in $\operatorname{SphK} 2^{-/}$mice [17]. These findings suggest that $\mathrm{SphK} 1$, but not $\mathrm{SphK} 2$, is involved in the development of pulmonary hypertension in mice and possibly humans. The authors 
also used a pharmacological inhibitor of S1P synthesis, SKi (SKI-II, 4-[[4-(4chlorophenyl)-2-thiazolyl]amino]-phenol) [17], which inhibits both SphK1 and SphK2 activity. When administered to rats $(10 \mathrm{mg} / \mathrm{kg}$ body weight, $\mathrm{i} / \mathrm{p})$, SKi prevented the development of hypoxia-dependent pulmonary hypertension, as assessed by reduced RVSP, RVH, and pulmonary vascular remodeling [17]. Conversely, heterozygote mice for the Sgpll gene, encoding S1P lyase which irreversibly cleaves S1P, exhibited increased lung S1P levels and elevated RVSP and RVH, which led to increased hypoxic-dependent pulmonary vascular remodeling compared with wild type (WT) mice [17]. Such opposing regulation of S1P levels by either reducing SphK1 activity (decreased S1P levels) or S1P lyase activity (increased S1P levels) is consistent with S1P being a key mediator in regulating vascular remodeling associated with changes in the proliferation of human PASMC. Indeed, the over-expression of wild type SphK1, which is fully catalytically competent, was reported to enhance human PASMC proliferation whereas a dominant negative kinase inactive form of SphK1 (G82D mutation) reduced PASMC proliferation [17]. Furthermore, PASMC from $S p h K 1^{-/-}$mice exhibited lower proliferation rates in response to chronic hypoxia compared with cells from WT mice [17], suggesting that S1P promotes PASMC proliferation in this mouse model.

One possible molecular mechanism by which SphK1 might regulate human PASMC proliferation and therefore vascular remodeling, involves so-called 'inside-out' signaling [18] where S1P formed by SphK1 is released from human PASMC to act upon S1P receptors, thereby promoting proliferation in an autocrine manner [17] (Figure 1). This model is supported by evidence showing that siRNA knockdown of $\mathrm{S}_{1} \mathrm{P}_{2}$ expression or $\mathrm{S} \mathrm{P}_{2}$ antagonism with JTE-013 (which is also a competitive antagonist of $\mathrm{S}_{1} \mathrm{P}_{4}$ [19]) ablated the S1P-stimulated proliferation of human PASMC [17]. This report suggested that $\mathrm{S} 1 \mathrm{P} / \mathrm{S} 1 \mathrm{P}_{2}$ uses $\mathrm{ERK}-1 / 2$ and $\mathrm{STAT3}$ signaling to promote human PASMC 
proliferation [17], and which is significant as STAT3 activation has been shown to be involved in pro-survival and proliferative pulmonary vascular phenotypes associated with human PAH $[3,20,21]$. Finally, the $\mathrm{S} \mathrm{P}_{2 / 4}$ antagonist, JTE-013 (8 mg/kg body weight, $\mathrm{i} / \mathrm{p}$ ) prevented and reversed pulmonary hypertension in the hypoxic mouse model [17], thereby confirming that $\mathrm{S}_{1} \mathrm{P}_{2}$ is involved in $\mathrm{S} 1 \mathrm{P}$-stimulated vascular remodeling in this model.

Increased SphK1 and SphK2 protein expression together with elevated plasma S1P levels were also detected in remodelled pulmonary arteries of patients with idiopathic pulmonary arterial hypertension (IPAH) and this has been recapitulated in the Sugen5416/hypoxia/normoxia $(\mathrm{Su} / \mathrm{Hx} / \mathrm{Nx} \mathrm{PAH})$ rat model of occlusive pulmonary hypertension [22]. A role for SphK1 in this rat model was demonstrated using the selective SphK1 inhibitor, SLP7111228 (10 mg/kg administered i/p), which reduced plasma S1P levels and the percentage of occluded pulmonary arteries and increased the percentage of non-occluded pulmonary arteries. These results suggest that SphK1 promotes occlusive pulmonary arteriopathy in severe PAH. In addition, the increase right ventricular internal diameter (RVID) observed in $\mathrm{Su} / \mathrm{Hx} / \mathrm{Nx}$ PAH rats was decreased by the SphK1 inhibitor [22]. Improved cardiovascular function with the SphK1 inhibitor was also observed as evidenced by increased cardiac index (CI) and reduced total pulmonary artery resistance index (TPRI). However, this was not accompanied by reduced RVSP or RVH, and chronic SphK1 inhibition had no effect on systemic arterial pressure and heart rate [22]. This is accounted for by the authors as being due to a direct beneficial effect of the SphK1 inhibitor on maladaptive hypertrophy [22]. 


\section{RAGE and SphK1 and PAH}

Receptor for Advanced Glycation End-products (RAGE), which is a member of the immunoglobulin family, can activate STAT3 and induce down-regulation of peroxisomeproliferator-activated receptor gamma (PPAR $\gamma)$ and BMPR2 [2]. This results in increased vascular smooth muscle proliferation and development of a PAH phenotype [2]. RAGE is important because it is one of the most up-regulated genes in PAH [23]. Ligands of RAGE include S100A4 which belongs to the family of EF-hand calcium-binding proteins [24] and inhibition of RAGE reverses the PAH phenotype in monocrotaline- and Sugen-induced rodent models [2]. Moreover, treatment of rat mesanglial cells with low concentrations of advanced glycation end-products (AGE) results in increased SphK activity and S1P production that promotes cell proliferation [25]. Therefore, we propose that the ability of AGE to stimulate SphK and S1P formation might provide a mechanism by which RAGE could potentially use $\mathrm{S} 1 \mathrm{P}$ and $\mathrm{S}_{2} \mathrm{P}_{2}$ to promote STAT3 activation and thereby induce down-regulation of BMPR2 and PPAR $\gamma$, a key process in the development of the PAH phenotype [2]. This possibility requires further investigation.

\section{SphK1 and miR-124 and miR-21}

There is also interaction between miRNA and SphK1, thereby linking the enzyme with a major disease-forming mechanism in PAH. For instance, miR-124 inhibits migration and invasion of ovarian cancer cells by down-regulating SphK1 and reduces gastric cancer cell proliferation also by targeting $\operatorname{SphK1}[26,27]$. miR-124, which regulates NFATc1, CAMTA1 (calmodulin-binding transcription activator 1) and PTBP1 (polypyrimidine tract-binding protein 1) to reduce nuclear factor of activated T cells (NFAT)-dependent transcription, is down-regulated in human PASMCs and mouse lungs under hypoxic conditions [28]. This is significant, as overexpression of miR-124 inhibits human PASMC 
proliferation [28]. Therefore, down-regulation of miR-124 in PAH might provide an explanation for the increased expression of SphK1, and the related effects of the enzyme on the PAH phenotype. In addition, transforming growth factor- $\beta$-SphK1/S1P signaling involving $\mathrm{S}_{1} \mathrm{P}_{2}$ upregulates miR-21 to promote fibrosis [29]. Moreover, the inhibition of miR-21 prevents/reverses pulmonary hypertension in hypoxic-treated mice [30]. miR-21 also enhances human PASMC proliferation and down-regulates BMPR2 [30], thereby potentially linking SphK1/S1P via miR-21 with pulmonary hypertension.

\section{Sphingosine 1-phosphate and Vascular Smooth Muscle Contractility.}

There is substantial evidence to suggest that SphK1 and S1P also play a significant role in regulating vascular contractility. In this regard, the basilar artery from Sphk1 ${ }^{-/}$ mice exhibits weaker vasoconstriction in response to $\mathrm{KCl}$ or $\mathrm{S} 1 \mathrm{P}$ or $\mathrm{U} 46619$ (TxA2 receptor agonist) compared with the arteries of WT or Sphk $2^{-/-}$mice [31]. One mechanism by which S1P might induce contraction of vascular smooth muscle appears to involve regulation of store-operated calcium channels (SOC). Specifically, angiotensin II (Ang-II) (AT1A receptor-mediated) has been reported to induce calcium entry into rat vascular smooth muscle cells [32] and this involves an early inositol 1,4,5-trisphosphate ( $\left.\mathrm{IP}_{3}\right)$ dependent mobilisation of $\mathrm{Ca}^{2+}$ from intracellular stores, evidenced by inhibition of this phase of $\mathrm{Ca}^{2+}$ with the phospholipase $\mathrm{C}$ inhibitor, U73122. A second sustained extracellular $\mathrm{Ca}^{2+}$ influx through SOC occurs and this can be reduced by pharmacological inhibition of SphK1 or SOC (SOC inhibitor, SKF96365). The SOC inhibitor was not effective in vascular smooth muscle cells derived from $S p h K 1^{-/-}$mice suggesting that Sphk1 is an up-stream regulator of SOC [32]. Intracellular S1P was also reported to mediate an effect on SOC-dependent $\mathrm{Ca}^{2+}$ influx independently of S1P receptors as the $\mathrm{S}_{1} \mathrm{P}_{1 / 3}$ receptor antagonist (VPC23019) and $\mathrm{S} \mathrm{P}_{2 / 4}$ antagonist (JTE-013) failed to modify 
the response [33]. Taken together with other findings described above, S1P appears to act both extracellularly (via $\mathrm{S}_{2} \mathrm{P}_{2}$ ) and intracellularly (via SOC) to regulate blood pressure changes.

More recent studies have questioned the role of SphK1 and S1P in promoting vascular remodeling, while maintaining some agreement concerning vascular smooth muscle contractility. In this regard, chronic treatment with Ang-II increased SphK1 mRNA expression in thoracic and abdominal aorta and mesenteric arteries of mice and increased SphK1 protein expression was detected in aorta and mesenteric artery homogenates [6]. In addition, S1P levels were increased in the plasma of Ang-II-treated WT but not Sphk1 ${ }^{-/-}$ mice [6]. Of relevance, $S p h k 1^{-/-}$mice developed significantly less severe Ang II-induced hypertension and cardiac hypertrophy compared to WT mice, suggesting a role for SphK1 in Ang II-induced hypertension [6]. However, fibrotic changes in the heart as well as intima-media thickness of cardiac arteries were similar between WT and $S p h k 1^{-/-}$mice, suggesting that SphK1 is not involved in promoting vascular remodeling in this model [6]. These findings are consistent with the observation that the selective and potent SphK1 inhibitor, PF-543 (at $\mathrm{nM}$ concentrations) had no effect on vascular remodeling in a mouse hypoxic model of pulmonary hypertension [33]. Of note, chronic Ang-II-induced hypertensive $\operatorname{Sphk1}^{-/}$mice exhibit less aortic and mesenteric artery contractility in response to phenylephrine or noradrenaline, suggesting that SphK1 also functions downstream of $\alpha 1$ adrenoceptors [6]. In addition, $\mathrm{S}_{1} \mathrm{P}_{2}$ expression was increased in the thoracic aorta in this model of hypertension [6], thereby potentially linking changes in SphK1 expression with 'inside-out' signaling by $\mathrm{S} 1 \mathrm{P}$ at $\mathrm{S}_{1} \mathrm{P}_{2}$ receptors to promote vasoconstriction and increased blood pressure. Indeed, Ang II-induced contraction of

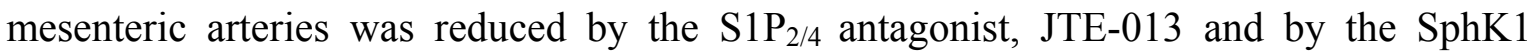
inhibitor, PF-543. The authors also showed lower cardiac hypertrophy and changes in 
aortic superoxide formation after Ang II infusion in $S p h k 1^{-/-}$mice; this is significant as superoxide can increase endothelial dysfunction [6]. In this regard, NADPH oxidase expression in the aorta and mesenteric arteries was increased in hypertensive $S p h k 1^{-/-}$mice compared with hypertensive WT mice [6].

There is also some controversy as to whether $\mathrm{S}_{1} \mathrm{P}_{2}$ regulates contractile tone. For instance, $\mathrm{S} 1 \mathrm{P}$ was reported to induce basilar artery contraction in rats and in $\mathrm{WT}$ and $\mathrm{S}_{1} \mathrm{P}_{2}$ null mice, but this was severely reduced in $\mathrm{S}_{1} \mathrm{P}_{3}$ null mice, suggesting a role for $\mathrm{S}_{1} \mathrm{P}_{3}$ rather than $\mathrm{S}_{2} \mathrm{P}_{2}$. In contrast, vasoconstriction in response to U46619 or to endothelin-1 was similar in vessels from $\mathrm{WT}, \mathrm{S}_{1} \mathrm{P}_{2}$ null and $\mathrm{S}_{1} \mathrm{P}_{3}$ null mice, suggesting that these receptors are not involved in the effect of U46619 and endothelin-1 [34]. In addition, this study revealed that JTE-013 could not only inhibit S1P-induced vasoconstriction, but also $\mathrm{KCl}-$, U46619- and endothelin-1-induced constriction, which persisted in $\mathrm{S}_{1} \mathrm{P}_{2}$ null mice [34] and which might suggest ablation of $\mathrm{S} \mathrm{P}_{4}$ signaling. The potential effect of the endothelium on vasoconstrictor response was demonstrated by use of the $\mathrm{S}_{1} \mathrm{P}_{1 / 3}$ receptor antagonist VPC23019, which enhanced S1P stimulated constriction, possibly as a consequence of blocking $\mathrm{S}_{1} \mathrm{P}_{1}$-induced nitric oxide generation in the endothelium. In this regard, S1P binding to $\mathrm{S}_{1} \mathrm{P}_{1}$ activates endothelial nitric oxide synthase (eNOS) in endothelial cells [36]. Finally, the $\mathrm{S} \mathrm{P}_{4}$ receptor agonist VPC23153 induced enhanced contraction of isolated pulmonary arteries from chronically hypoxic rats [36]. $\mathrm{S}_{1} \mathrm{P}_{4}$ was detected in both normotensive and hypertensive pulmonary arteries, suggesting that postreceptor signaling from $\mathrm{S}_{4} \mathrm{P}_{4}$ might be enhanced under hypertensive conditions, rather than changes in responsiveness being due to $\mathrm{S}_{1} \mathrm{P}_{4}$ expression. Since S1P-induced vasoconstriction was not reduced by VPC23019 or JTE-013 in this model, it appears that $\mathrm{S}_{1} \mathrm{P}_{2}$ and $\mathrm{S}_{1} \mathrm{P}_{3}$ do not play a role in the vasoconstrictor response of pulmonary vessels in rats [36]. 
In order to be able to translate these findings into the clinic, it is important that S1P receptor profiles of $\mathrm{PAH}$ patients be determined. Furthermore, identification and characterization of the S1P receptor involved in promoting vasoconstriction will be required. In our opinion, $\mathrm{S}_{1} \mathrm{P}_{2}$ is likely to be the key receptor, given that it is up-regulated in the thoracic aorta in mouse models of Ang II-stimulated pulmonary hypertension [6] and in patients with IPAH [17]. However, this hypothesis requires further investigation. $\mathrm{S}_{1} \mathrm{P}_{2}$ antagonists would need to be very selective for $\mathrm{S}_{1} \mathrm{P}_{2}$ and lack affinity for $\mathrm{S}_{1} \mathrm{P}_{1}$ or $\mathrm{S}_{1} \mathrm{P}_{3}$, as these latter receptors have been implicated in promoting endothelial function and vasodilation and therefore their blockade by an antagonist would potentially worsen hypertension. A summary of the role of rodent and human SphK1/S1P receptors in vascular smooth muscle contractility is presented in Figure 2.

There is also a role for S1P in other forms of hypertension. In this regard, sinusoidal vasoconstriction has an important role in portal hypertension and in this case, hepatic stellate cells function in a contractile manner promoted by S1P to enhance portal vein pressure in rat livers [37]. Administration of the $\mathrm{S}_{1} \mathrm{P}_{2 / 4}$ receptor antagonist, JTE-013 (1 $\mathrm{mg} / \mathrm{kg}, \mathrm{i} / \mathrm{v}$ ) to bile duct-ligated rats reduced portal vein pressure by $24 \%$, and had no effect in sham operated rats [37] or on mean arterial pressure in bile duct ligated rats. Rho kinase activity was increased in livers of bile duct-ligated rats and this was reduced by infusion of JTE-013 [37]. Moreover, ${\mathrm{S} 1 \mathrm{P}_{2}}_{2}$ mRNA expression was increased in livers of bile ductligated mice compared with sham-operated mice [37], suggesting that this receptor might mediate the effects of S1P. In addition, Rho kinase activity was reduced in $\mathrm{SIP}_{2}^{-/-}$mice subjected to bile duct ligation compared to wild type mice and the effects of the $\mathrm{S}_{1} \mathrm{P}_{2}$ antagonist were lost in the $S 1 P_{2}^{-/-}$mice. 


\section{SphK1 and Endothelial Cell Function}

S1P has an important role in the developmental of the endothelium and formation of blood vessels. Thus, VEGF promotes sprouting of endothelial cells to produce capillary tubes that are subsequently acted on by $\mathrm{S} 1 \mathrm{P} / \mathrm{S}_{1} \mathrm{P}_{1}$, which promotes stabilization of $\mathrm{VE}$ cadherin at endothelial junctions $[38,39]$. In addition. $\mathrm{S}_{1} \mathrm{P}_{1}$ responds to laminar shear stress to confer flow-dependent signaling in endothelial cells both in vitro and in vivo, and which stabilizes blood vessel development and homeostasis [40]. Significantly, S1P also exerts a protective role in endothelial cells by binding to $\mathrm{S}_{1} \mathrm{P}_{1 / 3}$ receptors to promote eNOS signaling [35].

The therapeutic potential of SphK1 inhibitors is likely to be compromised somewhat by the protective role of SphK1 against endothelial cell dysfunction. Thus, inhibition of endothelial SphK1 could potentially worsen endothelial dysfunction and thereby reduce the effectiveness of SphK1 inhibition in blocking vasoconstriction. The protective role of SphK1 is exemplified by studies using aortic rings isolated from Ang II-induced hypertensive $S p h k 1^{-/}$mice, which exhibit reduced vasorelaxation in response to acetylcholine compared to WT mice, suggesting that release of nitric oxide is impaired by loss of SphK1 [6]. In addition, low plasma S1P levels significantly correlated with impaired endothelial function in the Ang II-induced hypertensive mouse model [6]. Additionally, anandamide has been shown to induce relaxation of rat coronary arteries via an endothelium-dependent mechanism that involves $\mathrm{SphK} 1$ and $\mathrm{S}_{1} \mathrm{P}_{3}$ thereby providing additional evidence for activation of SphK1 and release of S1P from endothelial cells, which can then bind $\mathrm{S}_{3} \mathrm{P}_{3}$ in an autocrine manner to induce vasorelaxation [41]. Of note, genetic deletion of the Sphkl gene in Ang II-induced hypertensive mice had no significant effect on intima medial hypertrophy, suggesting that the observed impairment in 
endothelial function in $S p h k 1^{-/}$mice might explain why loss of this gene has no effect on vascular remodeling in this model [6].

Further evidence to support a role for SphK1 in protecting endothelial cell dysfunction has become evident in studies on Nogo B. Nogo-B inhibits serine palmitoyltransferase, which is the rate-limiting enzyme of de novo ceramide synthesis. Ceramide can be subsequently converted to sphingosine and S1P in mouse endothelial cells [42]. Indeed, mice deficient in Nogo B are hypotensive, resistant to angiotensin II-induced hypertension and exhibit preserved endothelial-dependent NO release [42]. This is accounted for, by

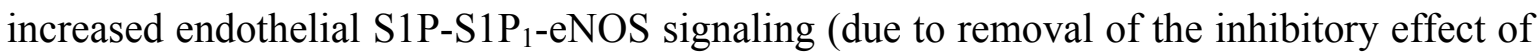
Nogo B on serine palmitoyltransferase), which promotes vasodilation. Indeed, this accounts for the hypotensive phenotype which can be reversed by pharmacological inhibition of serine palmitoyltransferase with myriocin [42]. In this regard, endothelial SphK1 is likely to play a significant role in producing $\mathrm{S} 1 \mathrm{P}$ to preserve endothelial cell function and to reduce vascular contractility and vascular remodeling. However, this hypothesis remains to be validated. A summary of the role of SphK1 in endothelial function is provided in Figure 2.

\section{Development of SphK1 Inhibitors for the Treatment of Pulmonary Arterial}

\section{Hypertension?}

The opposing functions of SphK1 in endothelial versus smooth muscle cells might allow extracellular factors that regulate the enzyme to instigate coordinated regulation and communication between the two cell types. However, the dual action of SphK1 might compromise the effect of SphK1 inhibitors in reducing blood pressure. In our opinion, more refined strategies that spare endothelial SphK1 function are required for translation of SphK1 inhibitors to the clinic for treatment of PAH. Human SphK1 exists as three N- 
terminal variants (SphK1a-c) encoded by the same gene [11]. Most studies have either focussed on SphK1a and/or have not defined the predominant isoform expressed in cell types studied. However, if these isoforms are differentially expressed in vascular smooth muscle and endothelial cells then one might be able to produce isoform sub-type-selective SphK1 inhibitors that prevent vascular remodelling/vasoconstriction and spare the endothelial protective mechanism(s). We propose that allosteric, rather than catalytic inhibitors might provide SphK1 isoform sub-type selectivity as these inhibitors could potentially target regulatory protein-protein interactions that exploit the $\mathrm{N}$-terminal variation of the SphK1 sub-types.

It may also be possible to exploit the two mechanisms by which SphK1 inhibitors affect SphK1 function. First, SphK1 inhibitors can rapidly and reversibly inhibit catalytic activity [8]. Second, SphK1 inhibitors induce a longer term (e.g. 24 h) ubiquitinproteasomal degradation of SphK1 thereby substantially reducing expression of the enzyme in human PASMC [7-9, 43]. Both mechanisms might impact contractility and/or vascular remodeling, although proteasomal degradation of SphK1 might predominantly affect remodeling, given the protective effect of Sphkl knockout on vascular remodeling in the hypoxic mouse model of pulmonary hypertension to reduce blood pressure and RV hypertrophy [17]. In addition, distinct SphK1 sub-types exhibit differential susceptibility to inhibitor-induced proteasomal degradation $[7,10]$ and we propose that this might be exploitable to preserve endothelial function, while inhibiting vasoconstriction. Further investigation in these areas is required to help to elucidate these hypotheses.

Certain SphK1 inhibitors can be considered 'direct' inducers of the proteasomal degradation of SphK1. For instance, PF-543 binds to SphK1 to induce a conformation that promotes its proteasomal degradation [9]. This is based on the strong correlation between the concentrations of inhibitor required to induce proteasomal degradation in human 
PASMC with the $\mathrm{K}_{\mathrm{i}}$ for inhibition of the catalysis of S1P formation by purified human SphK1 [43]. Another 'direct' SphK1 inhibitor, RB-005 (10 mg/kg i/v) [33] has been found to induce the proteasomal degradation of SphK1 in human PASMC in vitro and in pulmonary vessels from mice $(10 \mathrm{mg} / \mathrm{kg}, \mathrm{i} / \mathrm{v})$ exposed to normoxia, but failed to reduce SphK1 expression in the pulmonary vessels of mice treated with hypoxia for 3 weeks [33]. In addition, RB-005 had no significant inhibitory effect on RVP or vascular remodeling in hypoxic-treated mice [33], suggesting that the failure to remove SphK1 by proteasomal degradation from these hypoxic vessels prevents the inhibitor from reversing or blocking vascular remodeling. In addition, a weak inhibitor of SphK1, such as $\mathrm{SKi}$, has been shown to induce accelerated proteasomal degradation of SphK1 via activation of the proteasome in human prostate cancer cells [7]. This is an example of an 'indirect' inducer of the proteasomal degradation of SphK1. Indeed, we have shown that proteasomal degradation of SphK1 by 'indirect' inducers occurs via additional inhibition of dihydroceramide desaturase (Degs1) (an enzyme within the de novo ceramide synthesis pathway). In this regard, SKi inhibited Degs 1 activity in Jurkat and prostate cancer cells $[44,45]$. We have proposed that Degs 1 might function to limit protein degradation flux through the proteasome [44] and therefore its inhibition by SKi promotes proteasomal degradation of SphK1. Moreover, another study reported that SKi could prevent the development of hypoxic-dependent pulmonary hypertension in mice, as assessed by reduced RVSP, RVH and pulmonary vascular remodelling [17]. Therefore, it is possible that an 'indirect' inducer effect of SKi (i.e. via Degs1) on SphK1 degradation might bypass conformation-specific changes in SphK1 that could confer resistance to other 'direct' inducers (e.g. RB-005). This may explain why the previous study [17] was able to recapitulate the genetic loss of SphK1 using SKi, which led to reduced RVSP, RVH and 
pulmonary vascular remodeling in hypoxic mice. However, robust testing is warranted to further address these questions.

The resistance of SphK1 to 'direct' inducers as seen for RB-005 in hypoxic-treated mice [33], might also occur in other models of pulmonary hypertension, with observed differences in the modulation of vascular remodeling and blood pressure. For example, SphK1 is regulated by acetylation [46] and this can occur at the same lysine residues as ubiquitination. Aberrant acetylation of SphK1 in PAH could potentially confer resistance to SphK1 inhibitor-induced proteasomal degradation and is thus, worthy of further study.

The allosteric inhibitor of SphK1, $(S)$-FTY720 vinylphosphonate has been found to induce proteasomal degradation of human SphK1 [8], thereby producing an alternative conformation which appears to bypass resistance to proteasomal degradation in human androgen-independent prostate cancer cells [8]. A summary of the regulation of SphK1 by proteasomal degradation and the impact on pulmonary hypertension is presented in Figure 3. The major conclusion from the findings described above is that it is important to consider the effect of catalytic and allosteric SphK1 and Degs1 inhibitors on the ubiquitinproteasomal degradation status of SphK1 when evaluating the translational potential of these inhibitors for PAH. It is also important to establish whether SphK1 isoforms in human endothelial and vascular smooth muscle cells are differentially expressed as this might inform on targeting strategies designed to inhibit vasoconstriction/vascular remodeling and preserve endothelial function.

\section{SphK1 Inhibitors and Cardiac Hypertrophy}

Maladaptive hypertrophy leads to RV failure in the heart, the major cause of mortality in PAH patients [47]. Apoptosis has been shown to increase as RV function declines in monocrotaline treated rats [48] and pharmacologically-mediated reduction in RV 
hypertrophy has been associated with decreased apoptosis in monocrotaline treated rats, as evidenced by measurement of an increased Bcl2/Bax ratio [49]. Moreover, increased RV hypertrophy proceeds from and correlates with elevated RV pressure. However, sustained $\mathrm{RV}$ pressure can exist in the absence of significant RV hypertrophy in a persistent model of pulmonary hypertension in mice [51]. Administration of PF-543 (1 mg/kg, i/v) in the mouse hypoxic model of pulmonary hypertension has been shown to reduce RV hypertrophy (without changes in blood pressure), presumably via direct action on the heart, although this remains to be demonstrated [33]. In this study, cardiomyocyte apoptosis was observed in RV sections from chronic hypoxic mice, suggesting deleterious progression of RV [33]. Chronic administration of PF-543 to mice reduced the apoptotic index [33] suggesting that the SphK1 inhibitor might be protective. Hypoxia also induced formation of ADP-ribosylated PARP in the right ventricle, which was completely blocked by chronic administration of PF-543 to mice [33] and which supports evidence of a substantial blockade of apoptosis. Furthermore, the cardio-protective effect of PF-543 was associated with decreased pro-apoptotic p53 levels and increased pro-survival anti-oxidant nuclear factor (erythroid-derived 2)-like 2 (Nrf-2) expression in the right ventricle [33]. Indeed, pressure overload has been found to induce p53 expression via a mechanism involving the transcription factor, HIF $1 \alpha$; 553 , in turn, was reported to inhibit angiogenesis by reducing HIF1 $\alpha$ levels in a negative feedback manner in severe transverse aorta restriction in wild type and p53 deficient mice [51]. This finding is interesting because it suggests that PF-543 reduces p53 expression levels in the right ventricle, which may lead to angiogenesis/new blood vessel formation, in turn, increasing re-oxygenation to the heart. This might prevent development of maladaptive hypertrophy and subsequently apoptosis of cardiomyocytes. This hypothesis requires further investigation. 
Cardiac S1P levels have also been shown to increase in mice subjected to left anterior descending coronary ligation to induce post-myocardial infarction (MI). This is associated with increased $\mathrm{SphK} 1$ and $\mathrm{S}_{1} \mathrm{P}_{1}$ expression, suggesting that $\mathrm{MI}$ can regulate transcriptional activity of the genes encoding these proteins [52]. In this study administration of PF-543 $(1 \mathrm{mg} / \mathrm{kg}, \mathrm{i} / \mathrm{p})$ or FTY720 $(1 \mathrm{mg} / \mathrm{kg}, \mathrm{i} / \mathrm{p})$ reduced chronic cardiac inflammation and improved cardiac remodeling and dysfunction in vivo post-MI in mice [52]. FTY720 is converted to FTY720 phosphate, which is a $\mathrm{S}_{1} \mathrm{P}_{1}$ functional antagonist (see [11] for review) and FTY720 is also a SphK1 inhibitor [8]. These authors also showed that SphK1/S1P/S1P 1 -dependent STAT3 signaling and inflammation was modulated by FTY720, thereby reducing post-MI cardiac remodeling and dysfunction [52]. Therefore, SphK1 might participate in the chronic pro-inflammatory and fibrotic responses during the hypertrophic process that eventually leads to cardiomyocyte apoptosis and RV failure. Taken together with our findings on chronic hypoxic maladaptive hypertrophy, we propose that heart failure might be an additional target for therapeutic intervention with SphK1 inhibitors. However, these studies are preliminary, and further validation will be required prior to fully considering SphK1 as target to direct treatment of cardiac hypertrophy. A summary concerning the role of SphK1 in maladaptive hypertrophy is presented in Figure 4.

The detrimental role of SphK1 in long term cardiac remodeling contrasts with its protective role in ischemia/reperfusion where there is a loss of pre-conditioning or an exacerbation of injury in ex-vivo hearts from $S p h k 1^{-/}$and $S p h k 2^{-/}$mice $[53,54]$. In these models, ischemia promotes a rapid apoptotic/necrotic response that precedes longer term cardiac remodeling. In addition, genetic loss of SphK1 in mice results in poor resuscitation after cardiac arrest and reduced survival post-resuscitation, but this could be reversed by S1P lyase inhibition with 2-acetyl-4-(tetrahydroxybutyl)imidazole (THI) and 
was correlated with dihydroS1P rather than S1P levels [55]. These findings suggest a role for dihydroS1P in acute ischaemia [55]. The genetic loss of SphK1 and inhibition of S1P lyase results in alteration in $\mathrm{S}_{1} \mathrm{P}_{1}$ and $\mathrm{S}_{1} \mathrm{P}_{2}$ expression in the heart [55]. Such adaptive changes in S1P receptors as a possible consequence of altered S1P metabolism is an important cautionary consideration when evaluating findings from genetic 'loss of function' studies.

\section{Concluding Remarks}

There are a number of questions concerning the role of SphK1/S1P in PAH (see Outstanding Questions and Box 1). More emphasis should be placed on establishing functional interaction between SphK1 signaling pathways and other pathways linked with $\mathrm{PAH}$, such as BMPR2, RAGE and miRNA regulation. This approach might offer the possibility to bridge the experimental data with a solid rationale to support the use of SphK1 inhibitors in patients. We suggest that novel strategies will be necessary to produce inhibitors - presumably allosteric -- that induce a conformational change in SphK1 that is conducive to its proteasomal degradation and loss from human PASMC. Another approach might be to produce isoform sub-type SphK1 inhibitors that prevent vasoconstriction/vascular remodeling and hypertrophy but yet are able to preserve endothelial function to allow unimpeded vasodilation Alternatively, S1P receptor antagonists might represent a viable option for reversing vasoconstriction/vascular remodeling in pulmonary hypertension once the receptor (likely $\mathrm{S}_{1} \mathrm{P}_{2}$ ) involved in human disease has been identified. $\mathrm{S}_{1} \mathrm{P}_{2}$ antagonists might enable differentiation of action on PASMC versus endothelial cells. Finally, we propose that Degs1 might constitute an alternative target because its inhibition has been shown to indirectly promote the proteasomal degradation of SphK1 [44]. 
The SphK1 inhibitor, PF-543 also prevents maladaptive hypertrophy in a hypoxic mouse model of pulmonary hypertension [33] and cardiac remodeling post myocardial infarction [52]. Therefore, we hypothesize that SphK1 might represent an additional target for therapeutic intervention in cardiac hypertrophy, with SphK1 inhibitors acting directly in the heart to prevent heart failure. Further research should help elucidate the viability of these putative targets, and it will be exciting to follow up in these areas of lung and cardiac medicine.

\section{REFERENCES}

1. Benza, R.L. et al. (2012) An evaluation of long-term survival from time of diagnosis in pulmonary arterial hypertension from the REVEAL Registry. Chest 142, 448-456.

2. Meloche J. et al. (2013) Critical role for the advanced glycation end-products receptor in pulmonary arterial hypertension etiology. J. Am. Heart Assoc. 2, e005157.

3. Paulin R. et al. (2011) Signal transducers and activators of transcription-3/pim1 axis plays a critical role in the pathogenesis of human pulmonary arterial hypertension. Circulation. 123, 1205-1215.

4. Brock M. et al. (2009) Interleukin-6 modulates the expression of the bone morphogenic protein receptor type II through a novel STAT3-microRNA cluster 17/92 pathway. Circ Res. 104, 1184-1191.

5. Lane K.B. et al. (2000) Heterozygous germline mutations in BMPR2, encoding a TGFbeta receptor, cause familial primary pulmonary hypertension. Nat Genet. 26, 81-84.

6. Siedlinski, M. et al. (2017) Vascular transcriptome profiling identifies Sphingosine kinase 1 as a modulator of angiotensin II-induced vascular dysfunction. Sci. Rep. 7, 44131. 
7. Loveridge, C. et al. (2010) The sphingosine kinase 1 inhibitor 2-(p-hydroxyanilino)-4(p-chlorophenyl)thiazole induces proteasomal degradation of sphingosine kinase 1 in mammalian cells. J. Biol. Chem. 285, 38841-38852.

8. Tonelli, F. et al. (2010) FTY720 and (S)-FTY720 vinylphosphonate inhibit sphingosine kinase 1 and promote its proteasomal degradation in human pulmonary artery smooth muscle, breast cancer and androgen-independent prostate cancer cells. Cell. Signal. 22, 1536-1542.

9. Byun, H.S. et al. (2013) Novel sphingosine-containing analogues selectively inhibit sphingosine kinase (SK) isozymes, induce SK1 proteasomal degradation and reduce DNA synthesis in human pulmonary arterial smooth muscle cells. MedChemComm. $4(10)$.

10. Lim, K.G. et al. (2012) Inhibition kinetics and regulation of sphingosine kinase 1 expression in prostate cancer cells: functional differences between sphingosine kinase 1a and 1b. Int. J. Biochem. Cell Biol. 44, 1457-1464.

11. Pyne, S. and Pyne, N.J. (2011) Translational aspects of sphingosine 1-phosphate biology. Trends Mol. Med. 17, 463-472.

12. Spiegel, S. and Milstien, S (2003) Sphingosine-1-phosphate: an enigmatic signaling lipid. Nat. Rev. Mol. Cell Biol. 4, 397-407.

13. Blaho, VA. and Hla, T. (2014) An update on the biology of sphingosine 1-phosphate receptors. J. Lipid Res. 55, 1596-1608.

14. Hait, N.C. et al. (2009) Regulation of histone acetylation in the nucleus by sphingosine-1-phosphate. Science 325, 1254-1257.

15. Ahmad, M. et al. (2006) The effect of hypoxia on Lipid phosphate receptor and sphingosine kinase expression and mitogen activated protein kinase signaling in human pulmonary smooth muscle cells. Prostaglandins and other lipid mediators 79, 278-286. 
16. Schwalm, S. et al. (2008) Sphingosine kinase-1 is a hypoxia-regulated gene that stimulates migration of human endothelial cells. Biochem. Biophys. Res. Commun. $368,1020-1025$.

17. Chen, J. et al. (2014) The sphingosine kinase 1/sphingosine-1-phosphate pathway in pulmonary arterial hypertension. Am. J. Respir. Crit. Care Med. 190, 1032-1043.

18. Takabe, K. et al. (2008) 'Inside-out' signaling of sphingosine-1-phosphate: therapeutic targets. Pharmacol Rev. 60, 181-195.

19. Long, J.S. et al. (2010) Sphingosine 1-phosphate receptor 4 uses HER2 (ERBB2) to regulate extracellular signal regulated kinase-1/2 in MDA-MB-453 breast cancer cells. J. Biol. Chem. 285, 35957-35966.

20. Paulin, R. et al. (2012) STAT3 signaling in pulmonary arterial hypertension. JAKSTAT 1, 223-233.

21. Xu, W., and Erzurum, S.C. (2011) Endothelial cell energy metabolism, proliferation, and apoptosis in pulmonary hypertension. Compr. Physiol. 1, 357-372.

22. Gairhe, S. et al. (2016). Sphingosine-1-phosphate is involved in the occlusive arteriopathy of pulmonary arterial hypertension. Pulm. Circ. 6, 369-380.

23. Abdul-Salam, V.B. et al. (2010) Proteomic analysis of lung tissues from patients with pulmonary arterial hypertension. Circulation 122, 2058-2067.

24. Tarabykina, S. et al. (2007) Metastasis-associated protein S100A4: spotlight on its role in cell migration. Curr. Cancer Drug Targets. 7, 217-228.

25. Geoffroy, K. et al. (2004) Bimodal effect of advanced glycation end products on mesangial cell proliferation is mediated by neutral ceramidase regulation and endogenous sphingolipids. J. Biol. Chem. 279, 34343-34352.

26. Zhang, H. et al. (2013) MiR-124 inhibits the migration and invasion of ovarian cancer cells by targeting SphK1. J. Ovarian Res. 6:84. 
27. Xia, J. et al. (2012) miR-124 inhibits cell proliferation in gastric cancer through downregulation of SPHK1. J. Pathol. 227, 470-480.

28. Kang, K. et al. (2013) MicroRNA-124 suppresses the transactivation of nuclear factor of activated $\mathrm{T}$ cells by targeting multiple genes and inhibits the proliferation of pulmonary artery smooth muscle cells. J. Biol. Chem. 288, 25414-25427.

29. Liu, X. et al. (2016) Transforming growth factor- $\beta$-sphingosine kinase 1/S1P signaling upregulates microRNA-21 to promote fibrosis in renal tubular epithelial cells. Exp. Biol. Med. (Maywood). 241, 265-272.

30. Yang, S. et al. (2012) miR-21 regulates chronic hypoxia-induced pulmonary vascular remodeling. Am. J. Physiol. Lung Cell Mol. Physiol. 302:L521-L529.

31. Salomone, S. et al. (2010) Vessel-specific role of sphingosine kinase 1 in the vasoconstriction of isolated basilar arteries. Pharmacol. Res. 62, 465-474.

32. Wilson, P.C. et al. (2015) Inhibition of Sphingosine Kinase 1 Ameliorates Angiotensin II-Induced Hypertension and Inhibits Transmembrane Calcium Entry via StoreOperated Calcium Channel. Mol. Endocrinol. 29, 896-908.

33. MacRitchie, N. et al. (2016) Effect of the sphingosine kinase 1 selective inhibitor, PF543 on arterial and cardiac remodeling in a hypoxic model of pulmonary arterial hypertension. Cell Signal. 28, 946-955.

34. Salomone et al. (2008) Analysis of sphingosine 1-phosphate receptors involved in constriction of isolated cerebral arteries with receptor null mice and pharmacological tools. Br. J. Pharmacol. 153, 140-147.

35. Tölle, M. et al. (2016). Regulation of endothelial nitric oxide synthase activation in endothelial cells by $\mathrm{S} 1 \mathrm{P}_{1}$ and $\mathrm{S}_{\mathrm{P}} \mathrm{P}_{3}$. Biochem. Biophys. Res. Commun. 476, 627-634.

36. Ota, M. et al. (2011) $\mathrm{S} 1 \mathrm{P}_{4}$ receptor mediates $\mathrm{S} 1 \mathrm{P}$-induced vasoconstriction in normotensive and hypertensive rat lungs. Pulm. Circ. 1, 399-404. 
37. Kageyama, Y. et al (2012) Antagonism of sphingosine 1-phosphate receptor 2 causes a selective reduction of portal vein pressure in bile duct-ligated rodents. Hepatology 56, $1427-1438$.

38. Gaengel, K. et al. (2012) The sphingosine-1-phosphate receptor $\mathrm{S} 1 \mathrm{PR}_{1}$ restricts sprouting angiogenesis by regulating the interplay between VE-cadherin and VEGFR2. Dev. Cell 23, 587-599.

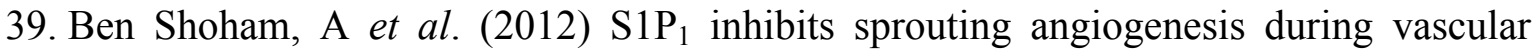
development. Development 139, 3859-3869.

40. Jung, B. et al. (2012) Flow-regulated endothelial S1P receptor-1 signaling sustains vascular development. Dev. Cell 23, 600-610

41. Mair, K.M. et al. (2010) Interaction between anandamide and sphingosine-1-phosphate in mediating vasorelaxation in rat coronary artery. Br. J. Pharmacol. 161, 176-192.

42. Cantalupo, A. et al. (2015) Nogo-B regulates endothelial sphingolipid homeostasis to control vascular function and blood pressure. Nat. Med. 21, 1028-1037.

43. Baek, D.J. et al. (2013) Synthesis of selective inhibitors of sphingosine kinase 1. Chem Comm (Camb). 49, 2136-2138.

44. McNaughton, M. et al. (2016) Proteasomal degradation of sphingosine kinase 1 and inhibition of dihydroceramide desaturase by the sphingosine kinase inhibitors, SKi or ABC294640, induces growth arrest in androgen-independent LNCaP-AI prostate cancer cells. Oncotarget 2016, 7, 16663-16675.

45. Venant, H. et al. (2015) The sphingosine kinase 2 inhibitor ABC294640 reduces the growth of prostate cancer cells and results in accumulation of dihydroceramides in vitro and in vivo. Mol. Cancer Ther. 14, 2744-2752.

46. Yu, H. et al. (2012) Acetylation of sphingosine kinase 1 regulates cell growth and cellcycle progression. Biochem. Biophys. Res. Commun. 417, 1242-1247. 
47. Vonk Noordegraaf, A. and Galiè, N. (2011) The role of the right ventricle in pulmonary arterial hypertension. Eur. Respir. Rev. 20, 243-253.

48. Campian, M.E. et al. (2009) Serial noninvasive assessment of apoptosis during right ventricular disease progression in rats. J. Nucl. Med. 50, 1371-1377.

49. Zuo, X.R. et al. (2012) Nicorandil prevents right ventricular remodeling by inhibiting apoptosis and lowering pressure overload in rats with pulmonary arterial hypertension. PLoS One. 7, e44485.

50. Dempsie, Y. et al. (2008). Converging evidence in support of the serotonin hypothesis of dexfenfluramine-induced pulmonary hypertension with novel transgenic mice. Circulation 117, 2928-2937.

51. Sano, M. et al. (2007) p53-induced inhibition of Hif-1 causes cardiac dysfunction during pressure overload. Nature 446, 444-448.

52. Zhang, F. et al. (2016) Sphingosine 1-phosphate signaling contributes to cardiac inflammation, dysfunction, and remodeling following myocardial infarction. Am. J. Physiol. Heart Circ. Physiol. 310, H250-61.

53. Jin, Z.Q. et al. (2008). Ischaemic postconditioning protects isolated mouse hearts against ischaemia/reperfusion injury via sphingosine kinase isoform-1 activation. Cardiovasc Res. 79, 134-140.

54. Vessey, D.A. et al. (2011). A sphingosine kinase form 2 knockout sensitizes mouse myocardium to ischemia/reoxygenation injury and diminishes responsiveness to ischemic preconditioning. Oxid. Med. Cell Longev. 2011:961059.

55. Gorshkova, I.A. et al. (2013) Inhibition of sphingosine-1-phosphate lyase rescues sphingosine kinase-1-knockout phenotype following murine cardiac arrest. Life Sci. 93, 359-366. 


\section{Figure legends}

Figure 1 The role of $\mathrm{SphK} 1 / \mathrm{S}_{1} \mathrm{P}_{2}$ in regulating pulmonary vascular remodeling in pulmonary hypertension. The schematic illustrates the role of $\mathrm{SphK} 1$ and $\mathrm{S}_{1} \mathrm{P}_{2}$ in regulating pulmonary arterial smooth muscle proliferation that contributes to vascular remodeling.

Figure 2 (Key Figure) The opposing roles of SphK1 in regulating vascular smooth muscle contractility (possibly via $\mathrm{S}_{2} \mathrm{P}_{2}$ ) and preservation of endothelial function (possibly via $\left.\mathrm{S} 1 \mathrm{P}_{1} / \mathrm{S} \mathrm{P}_{3}\right)$ that might suggest more nuanced treatment strategies of pulmonary hypertension with SphK1 inhibitors is necessary.

Figure 3 SphK1 inhibitors induce the ubiquitin-proteasomal degradation of SphK1 in PASMC via two distinct mechanisms (direct (PF-543) and indirect (SKi)). Direct SphK1 inhibitor-induced proteasomal degradation of SphK1 exhibits resistance under hypoxic conditions, while indirect inducers, such as SKi (which also inhibits Degs1) can reduce vascular remodeling under hypoxic conditions.

Figure 4 The role of SphK1 in regulating maladaptive hypertrophy via p53-dependent mechanism(s) in pulmonary hypertension. The SphK1 inhibitor, PF-543 reduces maladaptive hypertrophy and cardiomyocyte apoptosis in a hypoxic model of pulmonary hypertension. This is associated with a PF-543-induced reduction in ventricular p53 levels, which is significant as p53 decreases angiogenesis and thereby limits re-oxygenation of the heart. 


\section{Box 1. Clinician's Corner}

- There is potential for developing SphK1 inhibitors and S1P receptor antagonists for treatment of $\mathrm{PAH}$, but these might require more nuanced approaches to spare endothelial function while blocking vasoconstriction/vascular remodeling. The research is at a preclinical stage.

- Future direction should focus on the development of refined strategies to target SphK1, such as development of sub-type inhibitors of SphK1 and/or allosteric inhibitors designed to spare endothelial function and block/reverse vasoconstriction/vascular remodeling.

- Biomarker identification is required to determine target engagement and alteration of signaling networks that regulate vasoconstriction/vascular remodeling (e.g. S1P levels in the blood). This is essential, in order to enable stratification of patients groups that are more likely to be responsive to SphK1 inhibitors.

- Heart failure might be an additional target for therapeutic intervention with SphK1 inhibitors that act directly on the heart. Strong collaboration between clinical, basic and pharmaceutical scientists is required in order to accelerate the development of new S1P therapeutics for the treatment of PAH and heart failure. 


\section{Trends Box}

- SphK1 expression levels are increased in lungs from hypoxic mouse models of pulmonary hypertension and pulmonary arterial hypertensive patients.

- Increased SphK1 and SphK2 protein expression together with elevated plasma S1P levels are also increased in remodelled pulmonary arteries of patients with idiopathic pulmonary arterial hypertension and this has been recapitulated in a rat model of occlusive pulmonary hypertension.

- The SphK1 signaling pathway can regulate genes that are either up-regulated or down-regulated in PAH, such as RAGE, STAT3 and BMPR2, thereby identifying potential overlap with known PAH disease forming mechanisms.

- SphK1 is involved in hypoxic-dependent pulmonary hypertension, basilar artery contraction, Ang II-induced hypertension and subsequent cardiac hypertrophy.

- S1P formed by SphK1 might be released from arterial smooth muscle cells in an autocrine manner to promote proliferation via $\mathrm{S}_{1} \mathrm{P}_{2}$ receptors.

- SphK1 is protective against endothelial dysfunction. This might reduce effectiveness of SphK1 inhibitors in the treatment of PAH. More refined approaches to inhibiting SphK1 that enables selective targeting of the enzyme expressed in vascular smooth muscle is necessary.

- SphK1 can become resistant to ubiquitin-proteasomal degradation induced by SphK1 inhibitors in a hypoxic model of pulmonary hypertension. More nuanced approaches to inhibiting SphK1 that overcome resistance are needed.

- The SphK1 inhibitor, PF-543 prevents maladaptive hypertrophy in a hypoxic mouse model of pulmonary hypertension and cardiac remodeling post myocardial infarction suggesting an alternative utility for SphK1 inhibitors acting directly on the heart in heart failure. 


\section{Outstanding Questions}

- Which S1P receptors and SphK1 isoforms play a role in vasoconstriction and vasodilation in human pulmonary vessels and are these deregulated in PAH?

- What are the mechanisms by which SphK1 and S1P receptors regulate cellular signaling pathways involving RAGE, STAT3 and BMPR2 in humans and are these critical to the development of PAH?

- What is the major function of SphK1 in PAH with respect to its role in regulating vascular smooth muscle contractility, vascular remodeling and protecting against endothelial dysfunction?

- Is there a role for SphK1 in promoting inflammatory responses underlying changes in vascular function and remodeling in $\mathrm{PAH}$

- What is the mechanism that endows SphK1 with resistance to inhibitor-induced ubiquitin-proteasomal degradation in hypoxic induced pulmonary hypertension and is this evident in other models of PAH?

- Can refined strategies to target SphK1/S1P be developed to allow effective intervention in PAH?

- Can SphK1 inhibitors be developed that target different SphK1 sub-types to prevent/reverse vascular remodeling and hypertrophy but spare endothelial protective mechanism? 


\section{Glossary}

aortic ring: a transverse section of the aorta, used to measure vascular function in vitro

BMPR2: Bone morphogenetic protein receptor type 2 is a serine/threonine receptor kinase that binds bone morphogenetic proteins (BMPs), members of the transforming growth factor ligand family, and signals through intracellular SMAD proteins to activate transcription.

cardiac index (CI): a measure of cardiac function, calculated by dividing cardiac output by body weight.

Degs1: Dihydroceramide desaturase; an enzyme that catalyses the synthesis of ceramide from dihydroceramide.

HIF1a: Hypoxic inducible factor 1 alpha transcriptional factor is a master transcriptional regulator of genes in response to hypoxia.

HIF 2 $\alpha$ : Hypoxic inducible factor 2 alpha transcriptional factor.

hypoxic-induced pulmonary hypertensive: high blood pressure in the pulmonary arteries, arising due to a chronic deficiency of oxygen.

idiopathic pulmonary arterial hypertension (IPAH): an elevation of pulmonary artery pressure with no apparent cause.

'inside-out' signaling: the release of chemical signals from cells which then act in an autocrine (on the same cell) or paracrine (on a different cell) manner.

maladaptive hypertrophy: a pathological thickening of the heart wall that precedes heart failure. 
Nogo B: a protein of the endoplasmic reticulum which inhibits serine palmitoyltransferase (the rate-limiting enzyme of de novo ceramide biosynthesis) (also known as Reticulon 4B)

Nrf-2: Regulates transcription of anti-oxidant proteins that protect against oxidative stress.

occlusive pulmonary hypertension: hypertension arising from blockage of the pulmonary veins.

occlusive pulmonary arteriopathy: disease of the pulmonary arteries resulting in their blockage.

p53: tumor protein p53, which functions as a tumor suppressor.

PARP: poly (ADP-ribose) polymerase enzymes; involved in DNA repair and programmed cell death.

portal hypertension: high blood pressure in the hepatic portal system.

pressure overload: a pathological state where the heart muscle contracts against an excessive aortic pressure; chronic pressure overload can lead to cardiac hypertrophy.

RAGE: a transmembrane receptor for advance glycation end-products (glycated lipids and proteins) and damage-associated molecular pattern molecules.

right ventricular internal diameter (RVID): the inner diameter of the right ventricle.

right ventricular systolic pressure (RVSP): the peak of pulmonary artery pressure.

right ventricular hypertrophy (RVH): thickening of the right ventricle wall.

sinusoidal vasoconstriction: a reduction in the diameter of the sinusoidal blood vessels of the liver 
STAT3: a transcription factor that is a member of the Signal Transducer Activator of Transcription family; it is activated in response to various cytokines through Janus kinasecatalysed phosphorylation, followed by homo- or hetero-dimerisation.

Sugen5416/hypoxia/normoxia (Su/Hx/Nx PAH): an animal model of pulmonary hypertension that is induced by the combination of a vascular endothelial growth factor receptor antagonist, Sugen 5416 (SU5416) and chronic hypoxia.

total pulmonary artery resistance index (TPRI): a measure of pulmonary artery resistance, calculated by dividing RVSP by CI.

vascular remodelling: altered structure of resistance vessels giving rise to increased systemic vascular resistance in hypertension. 
Figure 1

SphK1 inhibition

Sphk1 ${ }^{-l-}$

Sphk1

$\longleftarrow$

$\longrightarrow$

(1) blood pressure

○ी $\begin{array}{lllllllllllllllllllllll}1 & 1 & 1 & 1 & 1 & 1 & 1 & 1 & 1 & 1 & 1 & 1 & 1 & 1 & 1 & 1 & 1 & 1 & 1 & 1 & 1\end{array}$

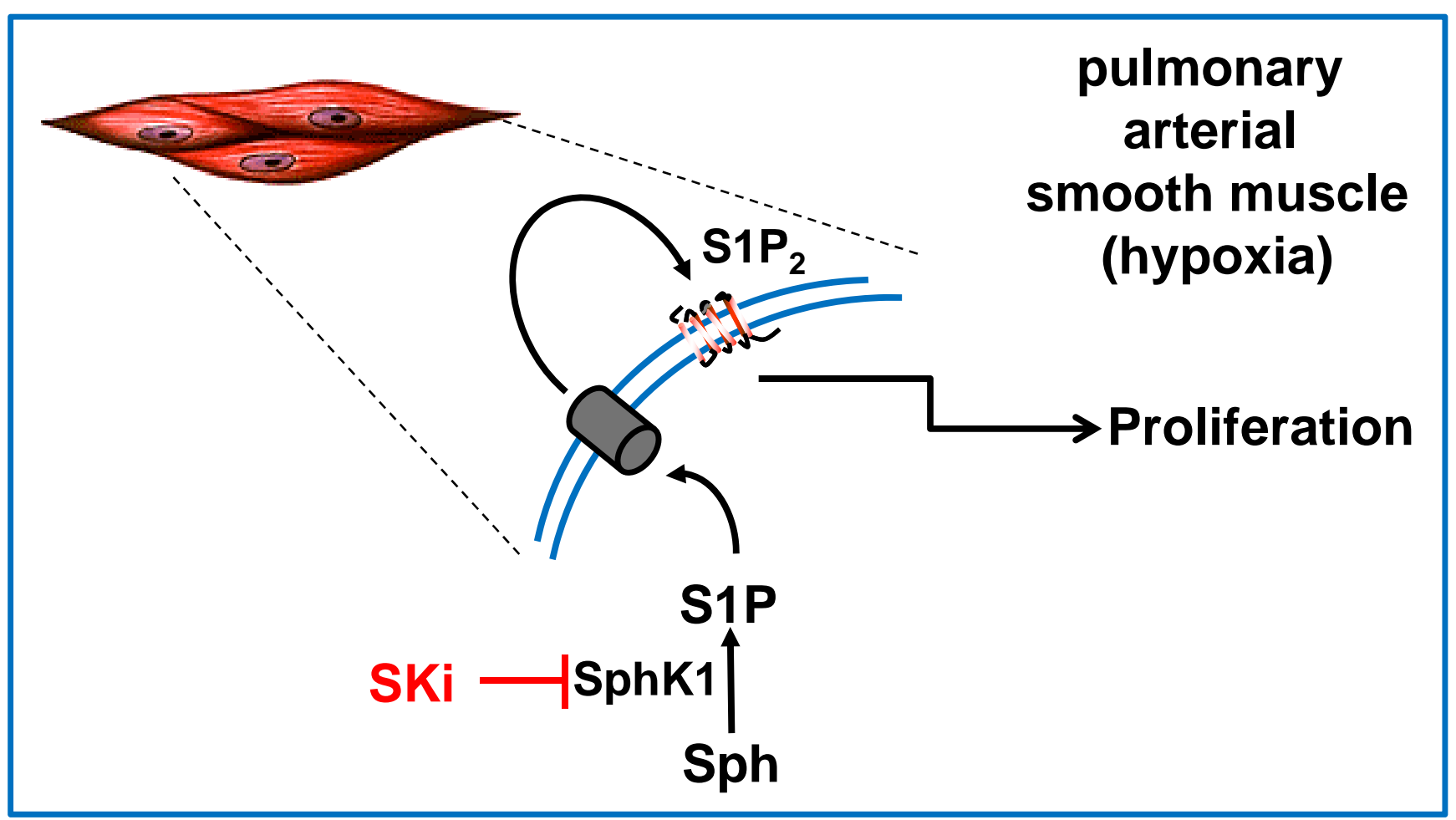


Figure 2
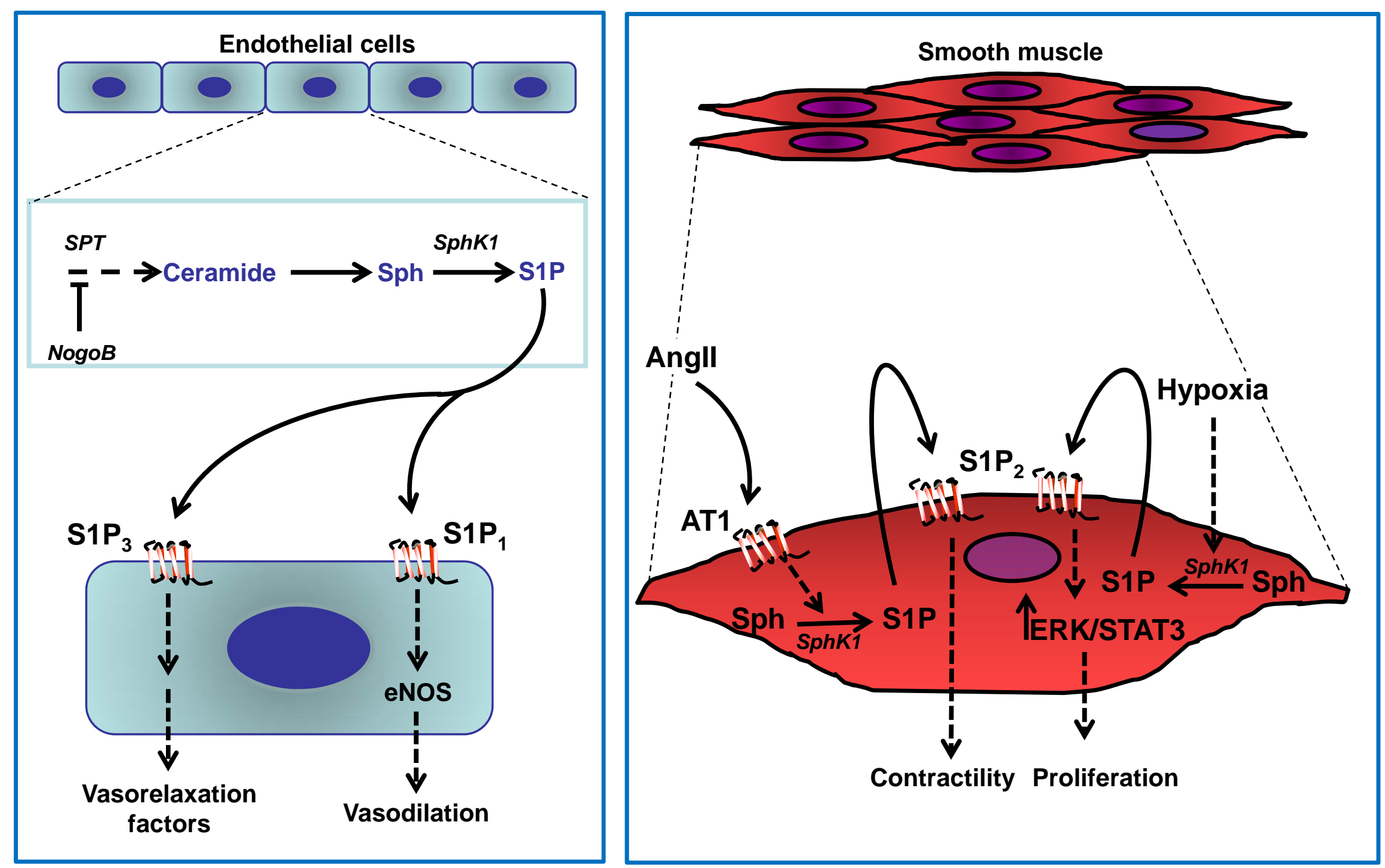
Figure 3

'Indirect'

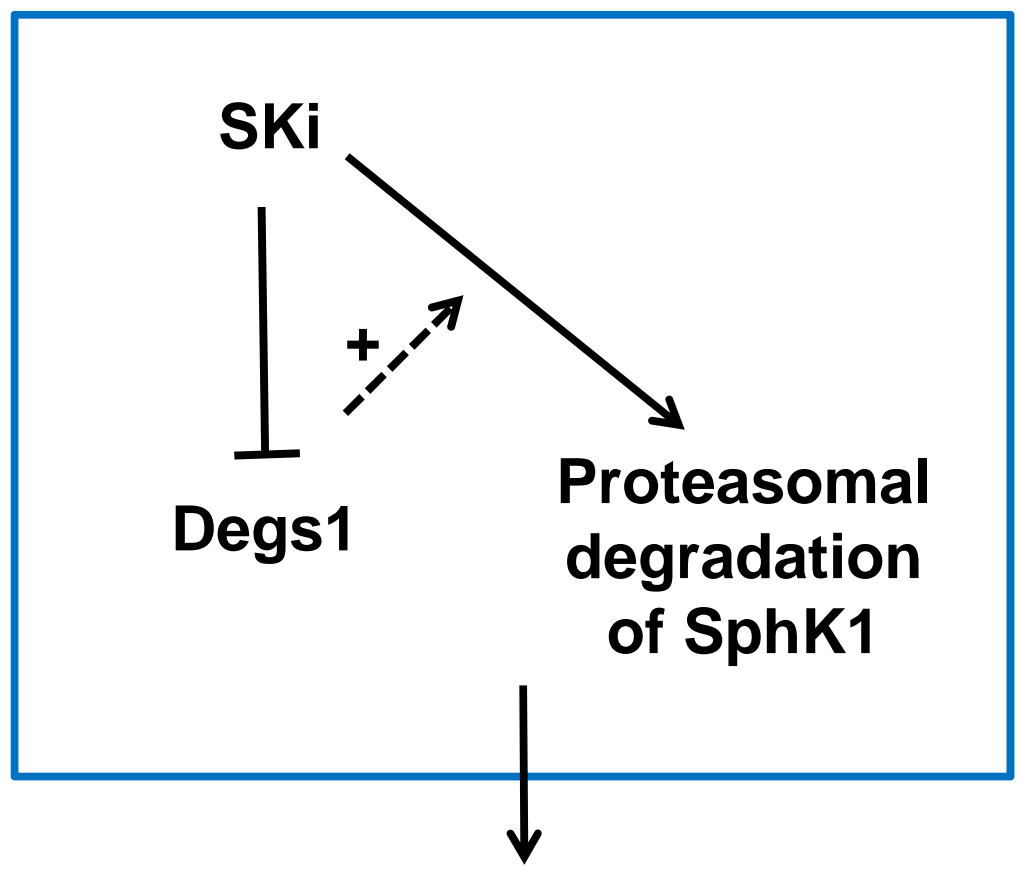

Reduced vascular remodelling and contractility

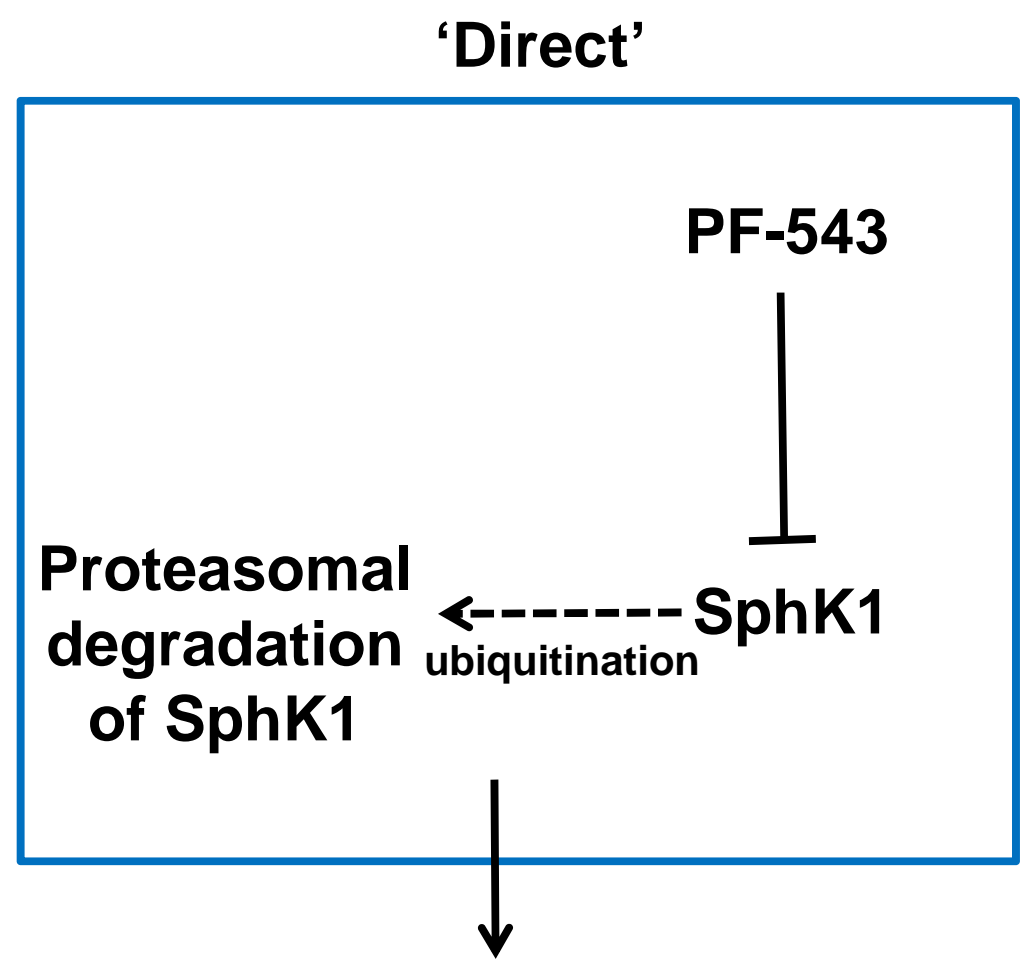

Resistance to proteasomal degradation of SphK1 and vascular remodelling under hypoxic conditions? 
Figure 4

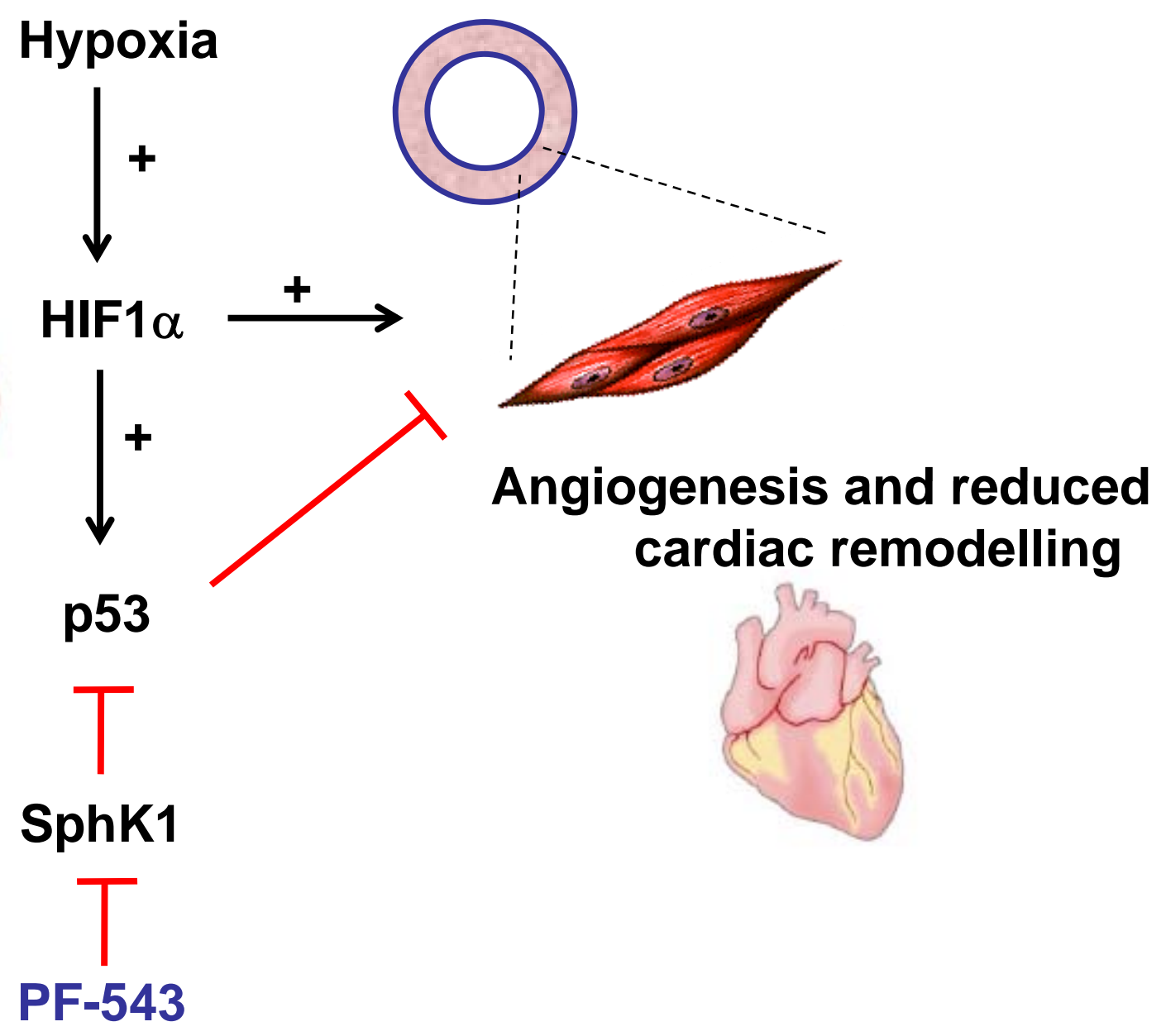

